\title{
Almost Sure Exponential Stability of Neutral Stochastic Hybrid Evolution
}

\section{Systems}

\author{
Hua Yang ${ }^{1}$, Feng Jiang2* \\ 1.School of Mathematics and Computer Science \\ Wuhan Polytechnic University \\ Wuhan 430023, China \\ School of Statistics and Mathematics \\ 2.Zhongnan University of Economics and Law \\ Wuhan 430073, China \\ e-mail: huay20@163.com; jeff20@163.com \\ *corresponding author
}

Keywords: mild solution; stochastic hybrid evolution systems; almost sure exponential stability

\begin{abstract}
This paper is mainly discussed almost sure exponential stability for mild solution of neutral stochastic hybrid evolution systems. Some conditions ensured the stability of mild solution of the systems are given. The earlier results are generalized and improved.
\end{abstract}

\section{Introduction}

The study of stochastic evolution systems has attracted the considerable attention, which are applied to many field such as chemistry, biology and physics [1,2]. At present, many dynamical behavior of the solutions of semilinear stochastic systems has been given. For example, [3] discussed exponential stability of mild solutions of stochastic partial systems with delays by fixed point theory. [4] studied approximate mild solutions of fractional stochastic evolution systems in Hilbert spaces.[5] studied evolution systems driven by general stochastic measures in Hilbert space.

On the other hand, in the real world, the systems often include the present state and the derivative of the state, which are described by neutral systems. Dynamic behavior of the neutral stochastic systems has been studied. [6] discussed existence and uniqueness of mild solutions to neutral stochastic partial functional systems under non-Lipschitz coefficients. [7] also discussed the existence of mild solutions of neutral semilinear stochastic functional systems under local nonLipschitz coefficients. [8] discussed exponential stability for semilinear neutral stochastic systems with delays by integral inequality. Luo et al. [9, 10] discussed stability for stochastic neutral partial differential systems by fixed point theory. [11] discussed asymptotic behavior for second order delay stochastic evolution systems. Besides, stochastic hybrid evolution systems drived by jumps have been also discussed extensively. [12] discussed exponential stability for neutral stochastic partial systems with jump. [13] discussed stability of mild solutions for second-order neutral stochastic evolution systems derived by Poisson jumps. Motivated by the above paper, in the paper we discussed almost sure exponential stability of neutral stochastic hybrid evolution systems by employing some integral inequalities.

The rest of the paper is organized as follows. In Section 2, mild definition and some useful lemmas are introduced. In Section 3, we establish almost sure exponential stability for neutral stochastic hybrid evolution systems. 


\section{Preliminaries}

Let $\Gamma$ and $\mathrm{Y}$ be two real, separable Hilbert spaces and $L(Y, \Gamma)$ be the space of bounded linear operators from $Y$ to $\Gamma$. For the sake of convenience, we shall use the same notation $\|\cdot\|$ to denote the norms in $\Gamma, \mathrm{Y}$ and $L(\mathrm{Y}, \Gamma)$ without any confusion. Let $(\Omega, \Xi, P)$ be a complete probability space equipped with some filtration $\Xi_{t}(t \geq 0)$ satisfying the usual conditions.

Let $L_{2}^{0}(\mathrm{Y}, \Gamma)$ denote the space of all $Q$-Hilbert-Schmidt operators $\sigma \in L(\mathrm{Y}, \Gamma)$. For the definition of an $\Gamma$-valued stochastic integral of an $L_{2}^{0}(Y, \Gamma)$-valued and $\Xi_{t}$-adapted predictable process with respectd to the $Q$-Wiener process $w(t)$, the readers can refer to [2]. $\tilde{N}(d v, d z)=N(d v, d z)-\lambda(d z) d v$, which is independent of $\mathrm{w}($.$) and is called the compensating martingale measure [1].$ Let $C([-\tau, 0], \Gamma)$ be the space of all right-continuous functions with left-hand limit $\varphi$. $C_{\Xi_{t}}^{b}=C_{\Xi_{t}}^{b}([-\tau, 0], \Gamma)$ be the family of all almost surely bounded, $\Xi_{t}(t \geq 0)$-measurable and $C([-\tau, 0], \Gamma)$ valued random variables. $-A$ is a closed, densely defined linear operator generating an analytic semigroup $S(t)(t \geq 0)$ on a separable Hilbert space $\Gamma$, then it is possible under some circumstances to define the fractional power $(-A)^{\alpha}:\left(D(-A)^{\alpha}\right) \rightarrow \Gamma[2]$.

Consider the following neutral stochastic hybrid evolution systems: for $t \in[0,+\infty)$

$$
\begin{gathered}
d[x(t)-D(t, x(t-\delta(t)))]=[A x(t)+f(t, x(t-\zeta(t)))] d t] \\
+g(t, x(t-\varsigma(t))) d w(t)
\end{gathered}
$$

$$
+\int_{Z} h(t, x(t-\eta(t)), z) \tilde{N}(d t, d z)
$$

with $x_{0}(\cdot)=\varphi \in C_{\Xi_{0}}^{v}$, where $\varphi$ is $\Xi_{0}$-meansurable and $\delta, \zeta, \quad, \eta:[0 .+\infty) \rightarrow[0, \tau](\tau>0)$ are bounded and continuous functions $f:[0,+\infty) \times \Gamma \rightarrow \Gamma, \quad g:[0,+\infty) \times \Gamma \rightarrow L_{2}^{0}(Y, \Gamma), \quad f:[0,+\infty) \times \Gamma \times U \rightarrow \Gamma$ are three suitable measurable mappings.

Definition 1. $x(t), t \in[-\tau,+\infty)$, is called a mild solution of the system (1), if $x(t)$ is a $\Xi_{t}(t \geq 0)$ adapted process and $x(t) \in \Gamma$ has a continuous paths on $t \in[0,+\infty)$ almost surely,

$$
\begin{aligned}
\mathrm{x}(t)= & S(t)(x(0)-D(0, x(-\delta(0))))+D(t, x(t-\delta(t))) \\
& +\int_{0}^{t} A S(t-s) D(s, x(s-\delta(s))) d s \\
& +\int_{0}^{t} S(t-s) f(s, x(s-\zeta(s))) d s \\
& +\int_{0}^{t} S(t-s) g(s, x(s-\varsigma(s))) d \omega(s) \\
& +\int_{0}^{t} \int_{Z} S(t-s) h(s, x(s-\eta(s)), z) \tilde{N}(d s, d z) .
\end{aligned}
$$

Lemma 1. If $0 \in \rho(-\mathrm{A})$, then for $\alpha \in(0,1]$

(i)There exist $M \geq 1$ and $\beta>0$ such that $\square S(t) h \llbracket \ll M e^{-\beta t} \square h \square, t \geq 0$, for any $h \in \Gamma$.

(ii) $\square(-A)^{\alpha} S(t) h \square M_{\alpha} e^{-\beta t} t^{-\alpha} \square h \square, t>0$, for any $h \in \Gamma$, where $M_{\alpha} \geq 1, \alpha \in(0,1]$.

Lemma 2. Let $\square\left(S(t) \llbracket M\right.$. For all $t \geq 0$ and let $\varphi:[0,+\infty) \rightarrow L_{2}^{0}$ be a predictable, $\Xi_{t}$-adapted process such that $\int_{0}^{t} E \square \varphi(s) \square_{L_{2}^{0}}^{p} d s<+\infty$ for some integer $p>2$ and any $t \geq 2$. Then, there exists a constant $c(p)>0$ such that for any fixed number $N>t_{0}$,

$$
\begin{aligned}
& E\left\{\sup _{N \leq t \leq N+1} \square \int_{N}^{t} S(t-s) \varphi(s) d \omega(s) \square^{p}\right\} \\
& \leq c(p) \int_{N}^{N+1} E \square \varphi(s) \square_{L_{2}^{0}}^{p} d s .
\end{aligned}
$$

Lemma 3. (Kunita's first inequality) For any $p \geq 2$, let $I(s)=\int_{0}^{t} \int_{Z} h(s, x) d \tilde{N}(d s, d x)$, there exists a constant $D(p)>0$ such that

$$
E\left(\sup _{0 \leq s \leq t}|I(s)|^{p}\right) \leq D(p)\left\{E\left(\int_{0}^{t} \int_{Z}|h(s, x)|^{2} \lambda(d x) d s\right)^{p / 2} \quad+E\left(\int_{0}^{t} \int_{Z}|h(s, x)|^{p} \lambda(d x) d s\right)\right\} .
$$


To obtain the stability of mild solutions to (1), we give the following conditions:

$\left(H_{1}\right)$ For any $x, y \in \Gamma$ and $t \geq 0$, there exist two positive constants $C_{1}, C_{2}>0$ such that $\square f(t, x)-f(t, y) \square<C_{1} \square x-y \square, \quad \square g(t, x)-g(t, y) \square_{L_{2}^{0}} \leq C_{2} \square x-y \square$,

$\int_{Z}|h(t, x, z)-h(t, y, z)|^{p} \lambda(d z) \vee\left(\int_{Z}|h(t, x, z)-h(t, y, z)|^{2} \lambda(d z)\right)^{p / 2}$

$\leq C_{3} \square x-y \square^{p}$,

and $f(t, 0)=g(t, 0)=h(t, 0, z)=0$.

$\left(H_{2}\right)$ For any $x, y \in \Gamma$ and $D(t, \cdot) \in D\left((-A)^{\alpha}\right)$, there exists a positive constants $C_{4}>0$ such that for $\alpha \in\left(\frac{1}{p}, 1\right](p \geq 2)\left\|(-A)^{\alpha} D(t, x)-(-A)^{\alpha} D(t, y)\right\| \leq C_{4}\|x-y\|, D(t, 0)=0, t \geq 0$,

$\left(H_{3}\right)$ For $\alpha \in\left(\frac{1}{p}, 1\right](p \geq 2), \kappa=\left\|(-A)^{-\alpha}\right\| C_{4}<1$.

Remark 1. Under $\left(H_{1}\right)-\left(H_{3}\right)$, (1) obviously has a trivial solution.

\section{Main results}

In this section, we will utilize some inequalities to derive some sufficient and verifiable conditions ensuring the almost sure exponential stability [3] for mild solution to (1). By [8, 13] we know that under $\left(H_{1}\right)-\left(H_{3}\right), \quad(1)$ is $p(\geq 2)$-moment exponential stable with some Lyapunov exponent $\mu$, i.e. $E\|x(t)\|^{p} \leq \bar{M} e^{-\mu t}$

Theorem 1. Supposed that the conditions $\left(H_{1}\right)-\left(H_{3}\right)$ are satistied with $p>2$, then the mild solution to (1) is almost sure exponential stable, i.e.

$$
\lim _{t \rightarrow+\infty} \frac{\log \|x(t)\|}{t} \leq-\frac{\mu}{2 p} \text {, a.s. }
$$

Proof . Let $\mathrm{N}$ be a sufficiently large positive integer and for $t \in[N, N+1]$, then

$$
\begin{aligned}
x(t) & =S(t-N)(x(N)-D(N, x(N-\delta(N)))) \\
& +D(t, x(t-\delta(t))) \\
& +\int_{N}^{t} A S(t-s) D(s, x(s-\delta(s))) d s \\
& +\int_{N}^{t} S(t-s) f(s, x(s-\zeta(s))) d s \\
& +\int_{N}^{t} S(t-s) g(s, x(s-\varsigma(s))) d \omega(s) \\
& +\int_{0}^{t} \int_{Z} S(t-s) h(s, x(s-\eta(s)), z) d \tilde{N}(d s, d z) .
\end{aligned}
$$

For arbitrary fixed $\varepsilon_{N}>0$, we have

$$
\begin{aligned}
& P\left\{\sup _{N \leq t \leq N+1}\|x(t)\|>\varepsilon_{N}\right\} \\
& \leq P\left\{\sup _{N \leq t \leq N+1}\|S(t-N)(x(N)-D(N, x(N-\delta(N))))\|>\frac{\varepsilon_{N}}{6}\right\} \\
& \left.+P\left\{\sup _{N \leq t \leq N+1} \| D(t, x(t-\delta(t)))\right) \|>\frac{\varepsilon_{N}}{6}\right\} \\
& \left.+P\left\{\sup _{N \leq t \leq N+1} \| \int_{N}^{t} A S(t-s) D(t, x(t-\delta(s)))\right) d s \|>\frac{\varepsilon_{N}}{6}\right\} \\
& \left.+P\left\{\sup _{N \leq t \leq N+1} \| \int_{N}^{t} S(t-s) f(s, x(s-\zeta(s)))\right) d s \|>\frac{\varepsilon_{N}}{6}\right\} \\
& \left.+P\left\{\sup _{N \leq t \leq N+1} \| \int_{N}^{t} S(t-s) g(s, x(s-\zeta(s)))\right) d \omega(s) \|>\frac{\varepsilon_{N}}{6}\right\} \\
& +P\left\{\sup _{N \leq t \leq N+1}\left\|\int_{N}^{t} S(t-s) h(s, x(s-\eta(s)), z) \tilde{N}(d s, d z)\right\|>\frac{\varepsilon_{N}}{6}\right\}
\end{aligned}
$$




$$
\begin{aligned}
& \leq\left(\frac{6}{\varepsilon_{N}}\right)^{p} E\left[\sup _{N \leq t \leq N+1}\|S(t-N)(x(N)-D(N, x(N-\delta(N))))\|^{p}\right] \\
& \left.+\left(\frac{6}{\varepsilon_{N}}\right)^{p} E\left[\sup _{N \leq t \leq N+1} \| D(t, x(t-\delta(t)))\right) \|^{p}\right] \\
& \left.+\left(\frac{6}{\varepsilon_{N}}\right)^{p} E\left[\sup _{N \leq t \leq N+1} \| \int_{N}^{t} A S(t-s) D(s, x(s-\delta(s)))\right) d s \|^{p}\right] \\
& \left.+\left(\frac{6}{\varepsilon_{N}}\right)^{p} E\left[\sup _{N \leq t \leq N+1} \| \int_{N}^{t} S(t-s) f(s, x(s-\zeta(s)))\right) d s \|^{p}\right] \\
& \left.+\left(\frac{6}{\varepsilon_{N}}\right)^{p} E\left[\sup _{N \leq t \leq N+1} \| \int_{N}^{t} S(t-s) g(s, x(s-\varsigma(s)))\right) d \omega(s) \|^{p}\right] \\
& +\left(\frac{6}{\varepsilon_{N}}\right)^{p} E\left[\sup _{N \leq t \leq N+1}\left\|\int_{N}^{t} \int_{Z} S(t-s) h(s, x(s-\eta(s)), z) \tilde{N}(d s, d z)\right\|^{p}\right] \\
& =\sum_{i=1}^{5} I_{i}
\end{aligned}
$$

Note that $E\|x(t)\|^{p} \leq \bar{M} e^{-\mu t}$. By Lemma 1, we have $I_{1} \leq\left(\frac{6}{\varepsilon_{N}}\right)^{p} M^{p} \bar{M}\left(\frac{1}{(1-\kappa)^{p-1}}+\kappa e^{\mu \tau}\right) e^{-\mu N}$.

By $\left(H_{2}\right)$, we have $I_{2} \leq\left(\frac{6}{\varepsilon_{N}}\right)^{p} \kappa^{p} \bar{M} e^{\mu t} e^{-\mu N}$. By Lemma 1, Lemma 2, we have

$$
I_{3} \leq\left(\frac{6}{\varepsilon_{N}}\right)^{p} M_{1-\alpha}^{p} \bar{M} C_{4}^{p} \bar{M}(q \beta)^{p \alpha-p / q}(\Gamma(1-q(1-\alpha)))^{p / q} e^{\mu \tau-\mu N}, I_{4} \leq\left(\frac{6}{\varepsilon_{N}}\right)^{p} \frac{M^{p} \bar{M} C_{1}^{p} e^{\mu t}}{(q \beta)^{p / q}} e^{-\mu N}
$$

By Lemma 3, we have $I_{5} \leq\left(\frac{6}{\varepsilon_{N}}\right)^{p} M^{p} c(p) C_{2}^{p} e^{\mu \tau-\mu N}$ and $I_{6} \leq\left(\frac{6}{\varepsilon_{N}}\right)^{p} M^{p} D(p) C_{3} e^{\mu \tau-\mu N}$. Thus,

$$
P\left\{\sup _{N \leq t \leq N+1}\|X(t)\|_{H}>\varepsilon_{N}\right\}<K\left(\frac{5}{\varepsilon_{N}}\right)^{p} e^{-\mu N}
$$

where

$$
\begin{aligned}
& K=M^{p} \bar{M}\left(\frac{1}{(1-\kappa)^{p-1}}+\kappa e^{\mu \tau}\right)+\kappa^{p} \bar{M} e^{\mu \tau} \\
& +M_{1-\alpha}^{p} \bar{M} C_{4}^{p}(q \beta)^{p \alpha-p / q}(\Gamma(1-q(1-\alpha)))^{p / q} e^{\mu \tau} \\
& +\frac{M^{p} \bar{M} C_{1}^{p} e^{\mu t}}{(q \beta)^{p / q}}+M^{p} c(p) C_{2}^{p} e^{\mu \tau}+M^{p} C_{3} D(p) e^{\mu \tau}
\end{aligned}
$$

Letting $\varepsilon_{N}=e^{-\frac{\mu N}{2 p}}$, such that

$$
P\left\{\sup _{N \leq t \leq N+1}\|x(t)\|>e^{-\frac{\mu N}{2 p}}\right\} \leq 6^{p} K e^{-\frac{\mu N}{2}} \text {. }
$$

Consequently, from the Borel-Cantelli Lemma, there exists a $T(\omega)>0$, for all $t>T(\omega)$, we have $\|x(t)\|^{p} \leq e^{-\frac{m N}{2}}$, a.s. That is, $\lim _{t \rightarrow+\infty} \frac{\log \|x(t)\|}{t} \leq-\frac{\mu}{2 p}$, a.s. The proof is completed.

When $\mathrm{p}=2$, by Theorem 1 , we have the following result.

Corollary 1 . Suppose that all the conditons of Theorem 1 hold with with $\mathrm{p}=2$ Then the mild solution of (1) is almost sure exponentially stble.

If (1) has no neutral term, (1) becomes the following stochastic evolution systems with jump:

$$
\begin{gathered}
d x(t)=[A x(t)+f(t, x(t-\zeta(t)))] d t+g(t, x(t-\varsigma(t))) d w(t) \\
+\int_{Z} h(t, x(t-\eta(t)), z) \tilde{N}(d t, d z)
\end{gathered}
$$

Similar to Theorem 1, we have

Corollary 2. Suppose that all the conditions of Theorem 1 hold. Then the mild solution of the above system without neural term is almost sure exponentially stble.

Remark 2: If (1) has no jump term, (1) becomes neural stochastic evolution systems. From Theorem 1, we can also obtain the system without jump is almost sure exponentially stable. 


\section{Conclusions}

In this paper, we have investigated almost sure exponential stability issue of neutral stochastic hybrid evolution systems. To establish the criteria of almost sure exponential stability, we employ some useful integral inequalities and Borel-Cantelli Lemma. The result is generalized some earlier results.

\section{Acknowledgment}

The work is supported by the Science and Technology Research Project of Hubei Provincial Department of Education (Q20151705).

\section{References}

[1] D. Applebaum, Levy Processes and Stochastic Calculus, second ed. Cambridge University Press, 2009.

[2] G. Da Prato and J. Zabczyk, Stochastic equations in infinite dimensions, Cambridge University Press, 1992.

[3] J. Luo, "Fixed points and exponential stability of mild solutions of stochastic partial differential equation with delays,” J. Math. Anal. Appl., vol. 342, pp. 753-760, 2008.

[4] J. Wang, "Approximate mild solutions of fractional stochastic evolution equations in Hilbert spaces,” Appl. Math. Comput., vol. 256, pp. 315-323, 2015.

[5] V. M. Radchenko, "Evolution equations driven by general stochastic measures in Hilbert space,” Theory of Probability and Its Appl., vol. 59, pp. 328-339, 2015.

[6] J. Jiang, Y. Shen, "A note on the existence and uniqueness of mild solutions to neutral stochastic partial functional differential equations with non-Lipschitz coefficients," Comput Math Appl., vol. 61, pp. 1590--1594, 2011.

[7] F. Jiang, "Mild solutions of neutral semilinear stochastic functional dynamic systems with local non-Lipschitz coefficients,” Adv. Math. Physics, vol. 2013, pp. 82353, 2013.

[8] H. Chen, "Integral inequality and exponential stability for neutral stochastic partial differential equations with delays,” J. Ineq. Appl., vol. 2009, pp. 297478, 2009.

[9] J. Luo, "Exponential stability for stochastic neutral partial functional differential equations,” J. Math. Anal. Appl., vol. 355, pp. 414-425, 2009.

[10] J. Luo, T. Taniguchi, Fixed points and stability of stochastic neutral partial differential equations with infinite delays,” Stoch. Anal. Appl., vol. 27, pp. 1163-1173, 2009.

[11] F. Liang, Z. Guo, "Asymptotic behavior for second order stochastic evolution equations with memory,” J. Math Anal. App., vol. 419, pp. 1333-1350, 2014.

[12] J. Cui, L. Yan, X. Sun, "Exponential stability for neutral stochastic partial diferential equations with delays and Poisson jumps,” Statist. Probab. Lett., vol. 81, pp. 1970--1977, 2011.

[13] R. Sakthivel, Y. Ren, "Exponential stability of second-order stochastic evolution equations with Poisson jumps,” Comm. Nonlinear Sci Numer. Simul., vol. 17, pp. 4517-4523, 2012. 